Quim. Nova, Vol. 28, No. 4, 716-718, 2005

\title{
DETERMINAÇÃO DE BROMATO EM MELHORADORES DE FARINHA POR CROMATOGRAFIA DE TROCA IÔNICA COM DETECÇÃO ESPECTROFOTOMÉTRICA
}

\author{
Rogério Marcos Dallago* e Irajá do Nascimento Filho \\ Centro Tecnológico, Curso de Química, Universidade Regional Integrada do Alto Uruguai e Missões, \\ Av. Sete de Setembro, 1621, 99700-000 Erechim - RS \\ Renato Zanella e Aline Machado Maroneze \\ Departamento de Química, Universidade Federal de Santa Maria, 99105-900 Santa Maria - RS
}

Recebido em 5/7/04; aceito em 5/11/04; publicado na web em 28/2/05

\begin{abstract}
BROMATE DETERMINATION IN FLOUR IMPROVERS BY ION EXCHANGE CHROMATOGRAPHY WITH SPECTROPHOTOMETRIC DETECTION. $\mathrm{KBrO}_{3}$ is registered by the FAO/OMS as a genotoxic and carcinogenic compound. In spite of this, $\mathrm{KBrO}_{3}$ is still employed by Brazilian bakeries. Nowadays ion exchange chromatography (IEC) is the most rapid and trustful method for $\mathrm{BrO}_{3}^{-}$analysis. When at high concentrations, chloride ions can interfere in the $\mathrm{BrO}_{3}^{-}$analysis, if the detection is performed by electrical conductivity. On the other hand, spectrophotometric detection, presented here is based on the absorption of $\mathrm{BrO}_{3}$ - in the ultraviolet region (210 - $230 \mathrm{~nm}$ ) where the absortion of chloride ions is very low, thus making possible the qualitative and quantitative analysis of $\mathrm{BrO}_{3}^{-}$in flour improver samples.
\end{abstract}

Keywords: bromate; flour improver; ion exchange chromatography.

\section{INTRODUÇÃO}

A utilização do bromato de potássio $\left(\mathrm{KBrO}_{3}\right)$ como aditivo alimentar na indústria de panificação tem sido praticada desde o início do século, sendo a primeira patente registrada em 1915, como uma mistura de sais melhoradores de pães ${ }^{1}$. O seu emprego baseiase nas propriedades oxidantes. O bromato oxida os grupos sulfídricos (-SH) das proteínas gliadina e glutenina (chamadas formadoras de glúten) a grupos dissulfídricos (-SS-) por desidrogenação da cisteína. Esta reação favorece a formação da rede proteíca pelo entrelaçamento entre as proteínas anteriormente citadas, através de pontes disssulfeto. Assim, são obtidos pães com maior capacidade de retenção de gases (aumento do volume), umidade e melhor textura ${ }^{2}$.

No entanto, foram evidenciados efeitos danosos à saúde em animais de laboratório ${ }^{3}$. Um significativo aumento na incidência de carcinomas renais foi observado em ratos, de ambos os sexos, alimentados com bromato via água de alimentação ${ }^{3,4}$. Através de estudos toxicológicos in vivo e in vitro o Comitê Conjunto da FAO/ OMS de Peritos em Aditivos Alimentares (JECFA, 1992) considerou o bromato de potássio como sendo um carcinógeno genotóxico, e, portanto, impróprio para uso como aditivo em farinhas e pães ${ }^{5}$. No Brasil, a resolução no 15/70 de 16/09/70 da Comissão de Normas e Padrões para Alimentos (CNNPA) proíbe o uso de bromato de potássio em produtos de panificação. Mesmo assim, Mousinho et al. $^{6}$ verificaram a presença de bromato de potássio na maioria das amostras de pães analisadas na cidade de Manaus no ano de 1988. O mesmo foi observado por Reyes et ll $^{7}$ em 18 marcas de melhoradores de farinha comercializados no estado de São Paulo e na região Nordeste do Brasil. Estes resultados evidenciam a necessidade de desenvolvimento de trabalhos científicos voltados à vigilância sanitária, os quais devem estar assessorados por metodologias analíticas simples, rápidas e confiáveis.

*e-mail: dallago@uri.com.br
Diversos métodos instrumentais (espectrofotométricos ${ }^{8-10}$, gravimétricos ${ }^{11}$ e cromatográficos ${ }^{12-24}$ ) são citados na literatura como passíveis de serem empregados para análise de bromato. A escolha do método está diretamente vinculada às características da amostra, como tipo de matriz (composição química) e, principalmente, a concentração do bromato. Tradicionalmente, a Cromatografia de Troca Iônica (CTI) com detecção condutimétrica tem sido empregada para determinação de bromato ${ }^{13-18}$. No entanto, sua aplicação está diretamente vinculada à concentração de íons cloreto presentes na amostra. A interferência do cloreto pode ser contornada mediante o emprego de detectores espectrofotométricos, após derivatização póscoluna do bromato, com ácido bromídrico $(\mathrm{HBr})$, a íon tribrometo $\left(\mathrm{Br}_{3}^{-}-\lambda_{\text {máx. }} \text { em } 267 \mathrm{~nm}\right)^{19-21}$. Outros reagentes derivatizantes empregados são a fucsina ${ }^{12}$ e a o-dianisidina ${ }^{19,22}$. Ambos reagentes conduzem à formação de complexos coloridos estáveis, que absorvem intensamente em 520 e $450 \mathrm{~nm}$, respectivamente. A derivatização, uma etapa onerosa, tanto em tempo quanto em custo, pode ser contornada pela determinação direta do bromato, íon que apresenta, entre suas propriedades físico-químicas, capacidade de absorver sensivelmente energia na região ultravioleta do espectro.

O presente trabalho teve como objetivo demonstrar a potencialidade da CTI com detecção espectrofotométrica no ultravioleta ( $\lambda$ $=220 \mathrm{~nm}$ ), para determinação de bromato em melhoradores de farinhas contendo íons cloreto como contaminantes.

\section{PARTE EXPERIMENTAL}

\section{Reagentes e amostra}

Todos os reagentes empregados $\left(\mathrm{NaCl}, \mathrm{KBrO}_{3}, \mathrm{NaHCO}_{3} \mathrm{e}\right.$ $\mathrm{Na}_{2} \mathrm{CO}_{3}$ ) foram de grau analítico. A água utilizada foi purificada em sistema Milli Q.

A amostra investigada, denominada "Emulsão reforçada de farinhas panificáveis", também conhecida como "Óleo Emulsificante", encontra-se disponível comercialmente e nos foi fornecida por uma padaria de Santa Maria - RS. 
O estado físico da amostra (líquido), juntamente com sua solubilidade em solventes polares, proporcionou uma minimização das etapas destinadas à preparação da amostra para a análise. Durante o pré-tratamento a amostra foi diluída em fase móvel $\left(\mathrm{NaHCO}_{3}-\right.$ 1,7 mmol L-1 / $\mathrm{Na}_{2} \mathrm{CO}_{3}-1,8 \mathrm{mmol} \mathrm{L}^{-1}$ ) e filtrada (filtro de membrana $0,45 \mu \mathrm{m}$ ) no momento da injeção.

\section{Materiais e métodos}

Os espectros na região do ultravioleta $(190-300 \mathrm{~nm})$ das soluções contendo íons bromato $\left(12,8 \mathrm{mg} \mathrm{L}^{-1}\right)$ e íons cloreto $\left(3,5 \mathrm{mg} \mathrm{L}^{-1}\right)$, foram obtidos empregando-se um espectrofotômetro UV - 1601PC (Shimadzu).

As análises foram realizadas por CTI, utilizando-se um Cromatógrafo Shimadzu 10AD acoplado a dois sistemas de detecção: condutimétrico (CDD-6A) e espectrofotométrico $(\lambda=220 \mathrm{~nm})$. O volume de amostra injetado foi $20 \mu \mathrm{L}$. A separação analítica foi conduzida à temperatura ambiente, em uma coluna de troca aniônica (Dionex) HPIC-AS4A (250 x 4 mm d.i.). Para eluição da amostra foi empregada uma vazão da fase móvel de $1,5 \mathrm{~mL} \mathrm{~min}{ }^{-1}$. Quando do emprego da detecção por condutividade elétrica faz-se necessária a utilização de um sistema supressor do tipo membrana (Supressora ASRSI Dionex). Como solução regeneradora do sistema supressor foi utilizada uma solução de ácido sulfúrico $10^{-2} \mathrm{~mol} \mathrm{~L}^{-1}$. Os picos resultantes da análise foram integrados em relação a sua área, com um integrador Shimadzu C-R6A.

A quantificação foi efetuada por padronização externa, usandose a calibração com múltiplos pontos. As soluções padrões, entre 0,1 e $100,0 \mathrm{mg} \mathrm{L}^{-1}$, empregadas na confecção da curva analítica foram obtidas mediante diluições sucessivas da solução estoque (100,0 mg $\mathrm{L}^{-1}$ ), preparada pela diluíção de $10 \mathrm{mg} \mathrm{de} \mathrm{BrO}_{3}^{-}$em $100 \mathrm{~mL}$ de água purificada. Os ensaios foram efetuados em triplicata.

\section{RESULTADOS E DISCUSSÃO}

A amostra parcialmente diluída (1:10) foi inicialmente analisada por CTI com detecção por condutividade elétrica. O cromatograma resultante (Figura 1a) apresenta apenas um pico em 2,02 min, característico do cloreto em elevadas concentrações. Após sucessivas diluições da amostra (1:1.000) foi possível identificar a presença de dois picos na região onde anteriormente observa-se somente o sinal de cloreto, um parcialmente definido (apresentando-se como um "ombro") e outro pico definido ( $t_{R}=1,91 \mathrm{~min}$ ), o qual foi vinculado ao cloreto (Figura 1b). Cromatogramas de soluções contento somente íons bromato (Figura 2a) ou íons cloreto (Figura 2b) demonstraram que estes íons apresentavam tempos de retenções muito próximos $\left(\mathrm{t}_{\mathrm{R} \text { (bromato) }}=1,79 \min\right.$ e $\mathrm{t}_{\mathrm{R} \text { (cloreto) }}=1,91 \mathrm{~min}$ ). Esta proximidade nos tempos de retenção dificulta a obtenção de cromatogramas com picos bem resolvidos (sem sobreposição de sinais) quando ambos íons encontram-se presentes na amostra, mesmo em baixas concentrações $\left(<3,0 \mathrm{mg} \mathrm{L}^{-1}\right)$ (Figura 1c). O cromatograda obtido para a amostra sintética, composta por uma mistura de íons bromato e cloreto (Figura 1c), apresentou-se muito similar ao cromatograma obtido para a amostra diluída 1.000 vezes (Figura 1b). Esta similaridade entre os dois cromatogramas, sugere que o "ombro" lateral observado na amostra diluída (Figura 1b) se deva à presença de íons bromato. Visando contornar este problema, ou seja, minimizar a interferência dos íons cloreto, permitindo obter um cromatograma com picos definidos (em relação ao bromato) e, conseqüentemente, uma análise mais confiável, fez-se a substituição do detector de condutividade por um detector espectrofotométrico.

O emprego do detector espectrofotométrico baseou-se nos espectros de absorção obtidos para os íons bromato e cloreto (Figura

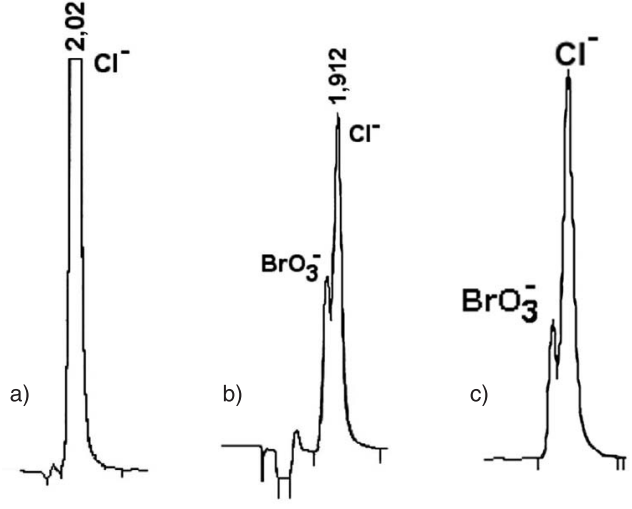

Figura 1. Cromatogramas referentes à amostra (a) diluída 10 vezes, (b) diluída 1.000 vezes e (c) amostra sintética $\mathrm{BrO}_{3}^{-} / \mathrm{Cl}$. Detector de condutividade elétrica

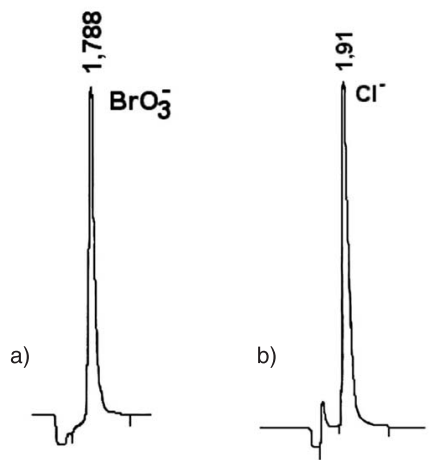

Figura 2. Cromatogramas referentes a soluções de (a) $\mathrm{BrO}_{3}^{-}\left(12,8 \mathrm{mg} \mathrm{L}^{-1}\right) e$ (b) $\mathrm{Cl}^{-}\left(3,5 \mathrm{mg} \mathrm{L}^{-1}\right)$. Detector de condutividade elétrica

3). O bromato apresenta uma banda de intensa absorção entre 205 e $225 \mathrm{~nm}$. Para o íon cloreto só se observa absorção abaixo dos $200 \mathrm{~nm}$. Esta diferença entre as regiões de absorção dos íons $\mathrm{Cl}^{-}(\lambda$ $<200 \mathrm{~nm}) \mathrm{e} \mathrm{BrO}_{3}^{-}(205 \mathrm{~nm}<\lambda<225 \mathrm{~nm})$ viabiliza a espectrofotometria na região ultravioleta do espectro como sistema de detecção para a análise de bromato em amostras contendo cloreto, independentemente da concentração deste na amostra.

De posse destes dados, a amostra diluida 1:1.000 foi analisada por cromatografia de troca iônica com detecção espectrofotométrica na região do ultravioleta $(220 \mathrm{~nm})$ (Figura 4). A análise foi conduzida nas mesmas condições experimentais (concentração, vazão e composição da fase móvel) utilizadas quando do emprego do sistema de detecção por condutividade (Figura 1b).

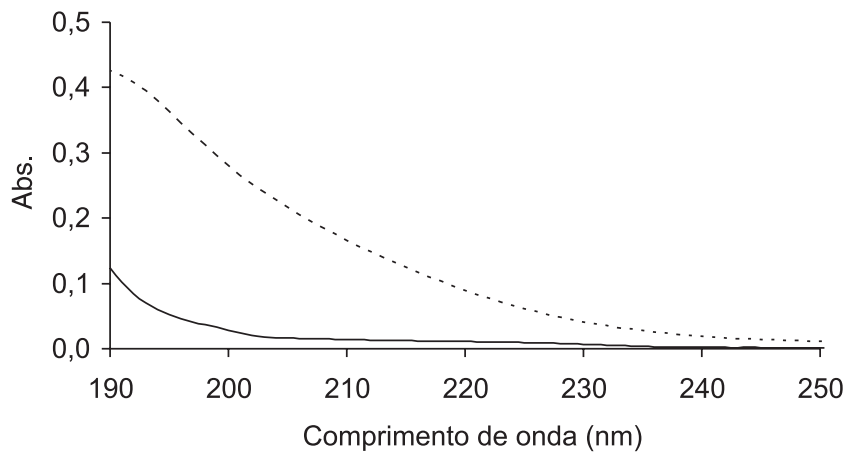

Figura 3. Espectros obtidos para as soluções de (---) bromato $\left(12,8 \mathrm{mg} \mathrm{L}^{-1}\right)$ e (_) cloreto $\left(3,5 \mathrm{mg} \mathrm{L}^{-1}\right)$ 
Tabela 1. Parâmetros utilizados para quantificação da amostra

\begin{tabular}{cccc}
\hline Equação da curva analítica & Coef. determinação $\left(\mathrm{r}^{2}\right)$ & LOD instrumental $\left(\mathrm{mg} \mathrm{L}^{-1}\right)^{*}$ & Faixa de linearidade $\left(\mathrm{mg} \mathrm{L}^{-1}\right)$ \\
\hline $\mathrm{y}=432.260 \mathrm{x}+231,3$ & 0,9998 & 0,05 & $0,1 \mathrm{a} 100,0$ \\
\hline
\end{tabular}

$\mathrm{x}=$ concentração em $\mathrm{mg} \mathrm{L}^{-1}$ e y = área do sinal; * quantidade mínima detectada pelo equipamento (sinal $\geq 3$ vezes ruído)

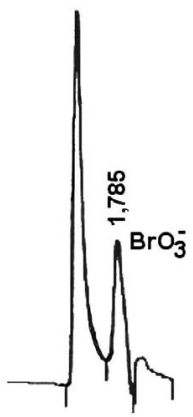

Figura 4. Cromatograma da amostra diluída 1.000 vezes. Detecção espectrofotométrica $(220 \mathrm{~nm})$

A detecção espectrofotométrica no ultravioleta proporcionou um cromatograma com picos bem resolvidos, onde o sinal do bromato aparece livre da interferência dos íons cloretos presentes na amostra permintido, desta forma, além da análise qualitativa, a realização da análise quantitativa de bromato. A quantificação foi efetuada pelo procedimento de padrões externos, usando-se a calibração com múltiplos pontos (Tabela 1). Para a faixa de concentração estudada $(0,1$ - 100,0 mg L $\left.{ }^{-1}\right)$ a curva analítica, representada pela equação da reta $(y=432.260 \mathrm{x}+231,3)$, apresentou uma excelente linearidade $\left(\mathrm{r}^{2}=\right.$ 0,9998). Para a amostra estudada, a concentração de bromato encontrada foi de aproximadamente $34.000,0 \mathrm{mg} \mathrm{L}^{-1}$, ou seja, $3,4 \%$ de sua composição. A determinação de bromato na amostra estudada empregando o método proposto forneceu resultados em boa concordância com os obtidos pelo método espectrofotométrico ${ }^{10}$. Os valores de bromato obtidos por ambos os métodos foram avaliados aplicando-se o teste $\mathrm{t}$ emparelhado.

$\mathrm{O}$ método, além de permitir a quantificação de bromato em amostras contaminadas com cloreto, apresentou excelentes parâmetros em relação à linearidade e, principalmente, ao limite de detecção do instrumento $\left(50 \mu \mathrm{g} \mathrm{L}^{-1}\right)$ (Tabela 1$)$, similar ao observado para a detecção condutimétrica $\left(5-25 \mu \mathrm{g} \mathrm{L^{-1 }}\right)$. Mediante prétratamentos adequados da amostra, como pré-concentração em fase sólida, o método poderá vir a atingir limites de detecção na ordem daqueles observados para os métodos com derivatização pós-coluna, ou seja, na faixa de 0,1 a $1 \mu \mathrm{g} \mathrm{L}^{-1} 24$.

Cabe ressaltar que o método proposto tem a vantagem de utilizar uma das colunas (AS4A) mais comumente utilizadas em cromatografia iônica, ampliando a capacidade de utilização do método proposto.

\section{CONCLUSÃO}

Os resultados obtidos neste trabalho demonstram a potencialidade do método de CTI com detecção espectrofotométrica, em relação à CTI com detecção condutimétrica para determinação do íon bromato em amostras contaminadas com elevadas concentrações de cloreto $\left(>3,5 \mathrm{mg} \mathrm{L}^{-1}\right)$. A CTI com detecção espectrofotométrica, além de proporcionar uma faixa de linearidade e limite de detecção similares à CTI com detecção condutimétrica, possibilita as análises qualitativa e quantitativa de bromato em melhoradores de farinha, não evidenciadas quando do emprego do detector condutimétrico.

Os resultados obtidos demonstram o potencial oferecido pela técnica, visando seu emprego rotineiro para auxiliar como ferramenta para uma política de vigilância sanitária, em relação à comercialização e emprego de produtos contendo bromato pela indústria panificadora brasileira.

A metodologia citada é passível de ser estendida para diferentes amostras com diferentes íons interferentes, desde que estes não absorvam radiação eletromagnética, nas regiões coincidentes com a do bromato, mais precisamente entre $210-230 \mathrm{~nm}$.

\section{AGRADECIMENTOS}

URI- Campus Erechim, FAPERGS e UFSM.

\section{REFERÊNCIAS}

1. Ranum, P.; Am. Assoc. of Cereal Chemists 1992, 37, 253.

2. Quaglia, G.; Ciencia y tecnologia de la panificación, Acribia: ZaragozaEspaña, 1991.

3. Kurokawa, Y.; Hayashi, Y.; Maekawa, A.; Takahashi, M.; Kokubo, T.; Odashima, S.; J. Nat. Cancer Inst. 1983, 71, 965.

4. Kurokawa, Y.; Maekawa, A.; Takahashi, T.; Hayashi, Y.; Environ. Health Persp. 1990, 87, 309.

5. World Health Organization (WHO); Evaluation of certain food additives and naturally occurring toxicants, Tech. Report Series, 1992, $\mathrm{n}^{\circ} 828$, $\mathrm{p}$. 29-30.

6. Mousinho, M. C.; Galvão, J. F.; Borborema, C.; Santos, M. A.; Anais do IV Encontro Nacional de Analistas de Alimentos, Belo Horizonte, Brasil, 1988.

7. Machado, M. C. M. S. T.; Reyes, F. G. R.; Rev. Farm. Bioquím. Univ. S. Paulo 1995, 31, 29.

8. Farrell, S.; Joa, J. F.; Pacey, G. E.; Anal. Chim. Acta 1995, 313, 121.

9. Xingguo, C.; Zhide, H.; Ketai, W.; Huitao, L.; Jian, H.; Food Chem. 2000, $20,509$.

10. Romele, L.; Achilli, M.; Analyst 1998, 123, 291.

11. Mendham, J.; Denney, R. C.; Barnes, J. D.; Thomas, M.; Vogel - Análise Química Quantitativa, 5ª ed., JC: Rio de Janeiro, 1992.

12. Achilli, M.; Romele, L.; J. Chromatogr., A 1999, 847, 271.

13. Gao, J.; Yoang, W.; Kang, J.; Hou, J.; Bian, H.; J. Chromatogr., A 1997, $789,195$.

14. Himata, K.; Noda, M.; Ando, S.; Yamada, Y.; Food Addit. Contam. 1997, $14,809$.

15. Kuldvee, R.; Kaljurand, M.; Smit, H. C.; J. Chromatogr., A 1997, 789, 247.

16. US EPA DBP/ICR Analytical Methods Manual, US Environ. Protection Agency, Cincinnati, OH, 1996.

17. Von Gunten, U.; Salhi, E.; Water Res. 1999, 33, 3239.

18. Gonçalves, M. E. P.; Camões, M. F.; Paiva, J. M.; Benoliel, M. J.; Carneiro, R. N.; Quim. Nova 2004, 27, 503.

19. Pepich, B. V.; Wagner, H. P.; Frebis, C.; Hautman, D. P.; Munch, D. J.; J. Chromatogr., A 2001, 920, 221.

20. Weinberg, H. S.; Yamada, H.; Anal. Chem. 1998, 70, 1.

21. Yamanaka, M.; Sakai, T.; Kumagai, H.; Inoue, Y.; J. Chromatogr., A 1997, $789,259$.

22. Wagner, H. P.; Pepich, B. V.; Hautman, D. P.; Munch, D. J.; J. Chromatogr., A 1999, 850, 119.

23. Willians, R. J.; Anal. Chem. 1983, 55, 851.

24. Mou, S.; Liu, Y.; Heberlig, S.; J. Chromatogr., A 2002, 956, 85. 